\title{
The weights of closed subgroups of a locally compact group
}

\author{
Salvador Hernández, Karl H. Hofmann and Sidney A. Morris
}

Communicated by Linus Kramer

\begin{abstract}
Let $G$ be an infinite locally compact group and let $\boldsymbol{\aleph}$ be a cardinal satisfying $\aleph_{0} \leq \aleph \leq w(G)$ for the weight $w(G)$ of $G$. It is shown that there is a closed subgroup $N$ of $G$ with $w(N)=\aleph$. Sample consequences are: (1) Every infinite compact group contains an infinite closed metric subgroup. (2) For a locally compact group $G$ and $\aleph$ a cardinal satisfying $\boldsymbol{\aleph}_{0} \leq \boldsymbol{\aleph} \leq w_{\text {loc }}(G)$, where $w_{\text {loc }}(G)$ is the local weight of $G$, there are either no infinite compact subgroups at all or there is a compact subgroup $N$ of $G$ with $w(N)=\aleph$. (3) For an infinite abelian group $G$ there exists a properly ascending family of locally-quasiconvex group topologies on $G$, say, $\left(\tau_{\boldsymbol{\aleph}}\right)_{\aleph_{0} \leq \boldsymbol{\aleph} \leq \operatorname{card}(G)}$, such that $\left(G, \tau_{\boldsymbol{\aleph}}\right)^{\wedge} \cong \hat{G}$.
\end{abstract}

\section{Introduction}

The weight $w(X)$ of a topological space $X$ is the smallest cardinal $\aleph$ for which there is a basis $\mathcal{B}$ of the topology of $X$ such that $\operatorname{card}(\mathscr{B})=\aleph$. A compact group $G$ is metric iff its weight $w(G)$ is countable, that is, $w(G) \leq \boldsymbol{\aleph}_{0}$. (See e.g. [9], A4.10ff., notably, A1.16.) In particular, all compact Lie groups are metric. It is not clear a priori that a compact group of uncountable weight contains an infinite closed metric subgroup. Indeed, in Proposition 5.6 below we will show that the precompact topological group defined on $\mathbb{Z}$ endowed with the Bohr-topology, which it inherits from its universal almost-periodic compactification, has no nonsingleton metric subgroup, while its weight is the cardinality of the continuum.

However, we shall prove the following theorem which, among other things, will show that every infinite compact group has an infinite metric subgroup.

Main Theorem. Let $G$ be a locally compact group of uncountable weight and let $\aleph_{0} \leq \boldsymbol{\aleph}<w(G)$. Then $G$ has a closed subgroup $N$ with $w(N)=\boldsymbol{\aleph}$.

In other words, for any infinite locally compact group $G$, the entire interval of cardinals $\left[\aleph_{0}, w(G)\right]$ is occupied by the weights of closed subgroups of $G$.

If $G$ is compact and connected, we shall see that $\left[\boldsymbol{\aleph}_{0}, w(G)\right]$ is filled even with closed normal and indeed connected subgroups. It remains unsettled whether a profinite group has, in this sense, enough normal closed subgroups. 
We shall deal with a proof of the Main Theorem in a piecemeal way.

For reasons of presenting a stepwise proof let us call $\mathcal{C}$ the class of all Hausdorff topological groups $G$ satisfying the following condition: for each infinite cardinal $\boldsymbol{\aleph} \leq w(G)$ there is a closed subgroup $H$ of weight $\boldsymbol{\aleph}$.

Our Main Theorem says that all locally compact groups are contained in $\mathcal{C}$.

We first aim to show that all compact groups are in $\zeta$ and we begin with compact abelian groups, proceed to compact connected, then to compact totally-disconnected groups and finally treat locally compact noncompact groups.

\section{Compact abelian groups}

For an abelian group $A$, let tor $A$ denote its torsion subgroup.

The first portion of our first observation is a consequence of a more precise statement due to W. R. Scott [13]. Since we prove what we shall need in a shorter way (and quite differently), we present a proof which will also establish the second part. A divisible hull of an abelian group we are using can be constructed in a very special way by using the results of E. A. Walker in [14].

Proposition 1.1. Let $A$ be an uncountable abelian group and $\boldsymbol{\aleph}_{0} \leq \boldsymbol{\aleph}<|A|$. Then $A$ contains a subgroup $B$ such that $(A: B)=\aleph$.

Moreover, if $(A: \operatorname{tor} A)$ is at least $\aleph$, then $B$ may be picked so that $B$ is pure and $A / B$ is torsion-free.

Proof. Let $D$ denote a divisible hull of $A$ according to [9, Proposition A1.33]. If $D=D_{1} \oplus D_{2}$ is any direct decomposition, and $\operatorname{pr}_{1}: D \rightarrow D_{1}$ is the projection onto the first summand of $D$, then $A /\left(A \cap D_{2}\right) \cong\left(A+D_{2}\right) / D_{2} \cong \operatorname{pr}_{1}(A)$. Now any nonzero subgroup $S$ of $D_{1}$ is a nonzero subgroup of the divisible hull of $A$ and therefore meets $A$ nontrivially (see [9, Proposition A1.33]), and thus we have $\{0\} \neq S \cap A \cap D_{1} \subseteq \operatorname{pr}_{1}(A)$; therefore $D_{1}$ is a divisible hull of $\operatorname{pr}_{1}(A)$. Hence either $\operatorname{pr}_{1}(A)$ is finite or else card $D_{1}=\operatorname{card} \operatorname{pr}_{1}(A)=\operatorname{card}\left(A /\left(A \cap D_{2}\right)\right)$ by [9, Proposition A1.33(i)].

As we control card $D_{1}$ by choosing $D_{1}$ appropriately, we aim to set $B=A \cap D_{2}$ and thereby prove our first assertion. We thus have to exclude the possibility that $\operatorname{pr}_{1}(A)$ might turn out to be finite by an inappropriate choice of $D_{1}$. We now let tor $A$ denote the torsion subgroup of $A$. Then tor $D$ is a divisible hull of tor $A$, and $D \cong(\operatorname{tor} D) \times(D /$ tor $D)$ by [9, p. 657, Proposition A1.38.]. We now distinguish two cases:

(a) Case $\operatorname{card}(\operatorname{tor} A)=\operatorname{card} A$. Since $A$ is uncountable, one of the $p$-primary components of tor $A$, as $p$ ranges through the countable set of primes, say $A(p)$, satisfies card $A(p)=\operatorname{card}(\operatorname{tor} A)=\operatorname{card} A$. In particular card $A(p)$ is uncount- 
able, that is, its $p$-rank card $A$ is uncountable and agrees with the $p$-rank of $D(p)$ (see $\left[9\right.$, p. 656, Corollary A1.36 (iii)]). In view of $D(p) \cong\left(\mathbb{Z}\left(p^{\infty}\right)^{(\operatorname{card} A)}\right)$ by [9, p.659, Theorem A1.42 (iii)], we can find a direct summand $D_{1}$ of $D(p)$ of $p$-rank $\aleph$, giving us a direct summand of tor $D$ and thus yielding a direct sum decomposition $D=D_{1} \oplus D_{2}$. Since the $p$-rank $\boldsymbol{\aleph}$ of $D_{1}$ is infinite, and $D_{1}$ is the divisible hull of $\operatorname{pr}_{1}(A)$, we know that $\operatorname{pr}_{1}(A)$ cannot be finite, whence

$$
\aleph=\operatorname{card} D_{1}=\operatorname{card}\left(A /\left(A \cap D_{2}\right)\right. \text {. }
$$

Our first assertion then follows with $B=A \cap D_{2}$.

(b) Case $\operatorname{card}(A / \operatorname{tor} A)=\operatorname{card} A$. Then the (torsion-free) rank of $D$ is card $A$ (see [9, p. 656, Corollary A1.36 (iii)]). By the structure theorem of divisible groups (see e.g. [9, Theorem A1.42]) and elementary cardinal arithmetic, we can write $D=D_{1} \oplus D_{2}$ with a torsion-free subgroup $D_{1}$ of cardinality $\aleph$. Then $\operatorname{pr}_{1}(A) \subseteq$ $D_{1}$ cannot be finite, and as in the first case, we let $B=A \cap D_{2}$ and have

$$
\aleph=\operatorname{card} D_{1}=\operatorname{card} A / B
$$

as in our first assertion.

It remains to inspect the case that $\operatorname{card}(A /$ tor $A) \geq \aleph$. Then we may assume tor $A \subseteq D_{2}$ and $D_{1}$ torsion-free. Since $A \cap D_{2}$ is torsion as $D_{2}$ is torsion, we have tor $A=A \cap D_{2}=B$. So, firstly, $A / B$ is torsion-free, and, secondly, $B$, as the torsion group of $A$ is pure in $A$.

The second part of the preceding proposition is also a consequence of [14, Theorem 4].

Corollary 1.2. Every uncountable abelian group has a proper subgroup of in$\operatorname{dex} \boldsymbol{\aleph}_{0}$.

(A different, but likewise not entirely trivial, proof by Hewitt and Ross is found in [7, p. 227].)

As usual, for a topological group $G$, the identity component of $G$ will be denoted $G_{0}$.

Corollary 1.3. Let $G$ be an infinite compact abelian group and assume $\boldsymbol{\aleph}_{0} \leq \boldsymbol{\aleph}<$ $w(G)$. Then $G$ has a closed subgroup $H$ with $w(H)=\boldsymbol{\aleph}$.

Moreover, if $w\left(G_{0}\right) \geq \boldsymbol{\aleph}$, then $H$ may be chosen to be connected.

Proof. By the Annihilator Mechanism ([9, Theorem 7.64]), for a subgroup $H$ of $G$ and its annihilator $H^{\perp}$ in $\hat{G}$, one has $\hat{H} \cong \hat{G} / H^{\perp}$ and thus (e.g. by [9, Theorem 7.76]) $w(H)=\operatorname{card} \hat{H}=\operatorname{card} \hat{G} / H^{\perp}$. Since card $\hat{G}=w(G)$, we have $\aleph<$ card $\hat{G}$.

Moreover, $H$ is connected iff $\hat{G} / H^{\perp}$ is torsion-free. (See [9, Corollary 8.5].) The corollary is therefore equivalent to Proposition 1.1 above. 
Let us see what we have in the case of $\boldsymbol{\aleph}=\boldsymbol{\aleph}_{0}$. A compact abelian group $A$ is metric iff $\hat{A}$ is countable ([9, Theorem 7.76]). Thus Corollary 1.3 trivially implies

Corollary 1.4. Every compact abelian group has an infinite closed metric subgroup.

A result by Efim Zelmanov says:

Theorem 1.5. An infinite compact group contains an infinite abelian subgroup.

Proof. See [15] or [12, p. 162].

This together with Corollary 1.4 implies

Corollary 1.6. Every compact group contains an infinite metric compact abelian subgroup.

While this corollary answers the question whether infinite compact groups have infinite compact metric subgroups in the affirmative, we should keep in mind that Zelmanov's theorem in itself is not a simple matter. It therefore appears worthwhile to pursue the question further.

\section{Connected compact groups}

The discussion of compact abelian groups yields at once the following information:

Corollary 2.1. Let $\boldsymbol{\aleph}$ be an infinite cardinal and let $G$ be a compact connected group with $w(G) \geq \aleph$. Then $G$ has a compact connected abelian subgroup $H$ with $w(H)=\boldsymbol{\aleph}$.

Proof. Let $T$ be a maximal compact connected abelian subgroup of $G$. Then we have $w(T)=w(G)$ by [9, Theorem 9.36(vi)]. Then the assertion follows from Corollary 1.3.

We see from Corollary 2.1 that for a compact connected group $G$ the set of all infinite cardinals $\leq w(G)$ is filled with the set of all infinite cardinals representing the weights of closed connected abelian subgroups. In the following we amplify this observation

Proposition 2.2. Let $\aleph$ be an infinite cardinal such that $\aleph<w(G)$ for a compact connected group $G$. Then $G$ contains a closed connected and normal subgroup $N$ such that $w(N)=\boldsymbol{\aleph}$. 
Proof. Following the Levi-Mal'cev Structure Theorem for Compact Connected Groups ([9, Theorem 9.24]), we have $G=G^{\prime} Z_{0}(G)$ where the algebraic commutator subgroup $G^{\prime}$ is a characteristic compact (see [9, Theorem 9.2]) connected semisimple (see [9, Corollary 9.6 and Theorem 9.19ff.]) subgroup, and the identity component of the center $Z_{0}(G)$ is a characteristic compact connected abelian subgroup.

Case 1. $w\left(G^{\prime}\right) \leq \aleph$. Then $w(G)=w\left(Z_{0}(G)\right)$, and by Corollary 1.3, $Z_{0}(G)$ contains a connected closed subgroup $N$ of weight $\aleph$; since it is central, it is normal.

Case 2. $w\left(G^{\prime}\right)>\boldsymbol{\aleph}$. If we find a compact connected normal subgroup $N$ of $G^{\prime}$, we are done, since the normalizer of $N$ contains both $G^{\prime}$ and the central subgroup $Z_{0}(G)$, hence all of $G=G^{\prime} Z_{0}(G)$. Thus it is no loss of generality to assume that $G=G^{\prime}$ is a compact connected semisimple group.

Case 2a. $G=\prod_{j \in J} G_{j}$ for a family of compact connected (simple) Lie groups. Then $w\left(G_{j}\right)=\boldsymbol{\aleph}_{0}$, and $\boldsymbol{\aleph}<w(G)=\max \left\{\boldsymbol{\aleph}_{0}\right.$, card $\left.J\right\}$ (see e.g. [9, EA4.3]). As $\boldsymbol{\aleph}$ is infinite and smaller than $w(G)$, we have $w(G)=$ card $J$. Then we find a subset $I \subseteq J$ such that $\operatorname{card} I=\boldsymbol{\aleph}$, and set $N=\prod_{i \in I} G_{i}$. Then $w(N)=\operatorname{card} I=\boldsymbol{\aleph}$.

Case 2b. Due to the Sandwich Theorem for Semisimple Compact Connected Groups $([9,9.20])$ there exists a family of simply-connected compact simple Lie groups $S_{j}$ with center $Z\left(S_{j}\right)$ and there are surjective morphisms

$$
\prod_{j \in J} S_{j} \stackrel{f}{\rightarrow} G \stackrel{q}{\rightarrow} \prod_{j \in J} S_{j} / Z\left(S_{j}\right)
$$

such that $q f$ is the product $\prod_{j \in J} p_{j}$ of the quotient morphisms

$$
p_{j}: S_{j} \rightarrow S_{j} / Z\left(S_{j}\right) \text {. }
$$

Now both products $\prod_{j \in J} S_{j}$ and $\prod_{j \in J} S_{j} / Z\left(S_{j}\right)$ have the same weight card $J$ which agrees with the weight of the sandwiched group $G$. Define $I$ as in Case 2a, set $N=f\left(\prod_{i \in I} S_{i}\right)$ and note that $q(N)=\prod_{i \in I} S_{i} / Z\left(S_{i}\right)$. Hence $N$ is sandwiched between two products with weight card $I=\boldsymbol{\aleph}$ and hence has weight $\boldsymbol{\aleph}$. This proves the existence of the asserted $N$ in the last case.

At this juncture we are faced with a crossroad between compact connected groups and profinite groups.

Lemma 2.3. Let $G$ be an arbitrary compact group. Then the following conclusions hold:

(i) $G$ and $G_{0} \times G / G_{0}$ are homeomorphic. In particular, if $G$ is infinite, then

$$
w(G)=\max \left\{w\left(G_{0}\right), w\left(G / G_{0}\right)\right\} .
$$


(ii) There is a profinite subgroup $D$ of $G$ such that $G=G_{0} D$ and $G_{0} \cap D$ is normal in $G$ and central in $G_{0}$. If $w\left(G_{0}\right)<w(G)$, then

$$
w(G)=w\left(G / G_{0}\right)=w(D) .
$$

(iii) If $G$ is profinite, then $G$ and $(\mathbb{Z} / 2 \mathbb{Z})^{w(G)}$ are homeomorphic.

Proof. For (i) see $[9,10.38]$. Regarding statement (ii), see $[9,9.41]$ and note that $G / G_{0} \cong D /\left(D \cap G_{0}\right)$. For (iii), see $[9,10.40]$.

After Lemma 2.3 the question whether a compact group $G$ is in $\mathcal{C}$ splits into two cases:

Case 1. If $w\left(G_{0}\right)=w(G)$, one may assume that $G$ is connected.

Case 2. If $w\left(G_{0}\right)<w(G)$, one may assume that $G$ is profinite.

\section{Profinite groups and the generating degree}

Now we have to reach beyond connectivity, all the while still staying within the class of compact groups.

We refer to a cardinal invariant for compact groups $G$ which is one of several alternatives to the weight $w(G)$, namely, the so-called generating degree $s(G)$ (see [9, Definition 12.15]). The definition relies on the Suitable Set Theorem, loc. cit. Theorem 12.11, which in turn invokes the so-called Countable Layer Theorem (see [8] or [9, Theorem 9.91]). Indeed recall that in a compact group $G$ a subset $S$ is called suitable iff it does not contain 1, is closed and discrete in $G \backslash\{1\}$, and satisfies $G=\overline{\langle S\rangle}$. The Suitable Set Theorem asserts that every compact group $G$ has a suitable set. A suitable set is called special iff its cardinality is minimal among all suitable subsets of $G$. The cardinality $s(G)$ of one, hence every, special suitable set is called the generating degree of $G$.

The relevance of the generating degree in our context is the following

Proposition 3.1. Let $G$ be a profinite group with uncountable weight. Then we have $w(G)=s(G)$.

Proof. By Proposition 12.28 of [9], for an infinite profinite, that is, compact totally disconnected group we have

$$
w(G)=\max \left\{\boldsymbol{\aleph}_{0}, s(G)\right\} .
$$

This implies the assertion immediately in the case of $w(G)>\boldsymbol{\aleph}_{0}$.

The next step, namely proving that every profinite group is in $\mathcal{C}$, will be facilitated by a lemma on suitable sets for which all ingredients are contained in [9]. 
Lemma 3.2. The following hold:

(a) Let $S$ be any suitable set of a topological group $G$. Then card $S \leq w(G)$.

(b) If $G$ is profinite and $S$ is an infinite suitable subset of $G$, then card $S=w(G)$.

Proof. (a) Let $\mathcal{B}$ be a basis of the topology of $G$ of cardinality $w(G)$. Since $S$ is discrete in $G \backslash\{1\}$, for every element $x \in S$ there is an element $U(x) \in \mathscr{B}$ with $U(x) \cap S=\{x\}$. Then $x \mapsto U(x): S \rightarrow \mathscr{B}$ is an injective function and thus card $S \leq \operatorname{card} \mathscr{B}=w(G)$. (Cf. [9, p. 620, proof of 12.16].)

(b) Assume that $G$ is profinite. If $w(G)$ is uncountable, then

$$
w(G)=s(G) \leq \operatorname{card} S
$$

by Proposition 3.1 and the definition of $s(G)$. From this and (a), $w(G)=\operatorname{card} S$ follows.

Now assume that $S$ is infinite and that $w(G)=\aleph_{0}$. Then

$$
\aleph_{0} \leq \operatorname{card}(S) \leq w(G)=\aleph_{0}
$$

by (a) above, and so $w(G)=\operatorname{card} S$ holds as well.

The significance of this lemma is that for a profinite group, infinite suitable subsets all have the same cardinality, namely, the weight of the group.

We are indebted to the referee for the observation that part (a) of Lemma 3.2 does not depend on compactness and thus remains intact for arbitrary topological groups in the face of the general definition of a suitable set in [9, Definition 12.1].

It is instructive to take note of the following remarks which are pertinent to this context:

Remark a. The universal monothetic and the universal solenoidal compact groups $G$ have generating degree $s(G)=1$, density $d(G)=\boldsymbol{\aleph}_{0}$ (i.e. they are separable), and weight $w(G)=2^{\aleph_{0}}$. (For the concept of density see e.g. [9, p. 620, Definition 12.15].)

Remark b. If $H$ is a precompact group whose Weil completion $G$ is profinite and if $H$ has an infinite relatively compact suitable subset, then card $H \geq w(H)$.

Proof. Assume that $S$ is an infinite relatively compact suitable subset of $H$. Then by [9, p. 616, Lemma 12.4], $S$ is a suitable subset of $G$ and by Lemma 3.2 it follows that card $H \geq \operatorname{card} S=w(G)=w(H)$.

Remark c. (i) For every compact group $G$ and cardinal number $\aleph$, the inequalities $\boldsymbol{\aleph}<w(G) \leq 2^{\aleph}$ imply $d(G)<w(G)$. 
(ii) Let $G$ be a compact group of weight $\boldsymbol{\aleph}_{1}$. Then $G$ contains countable dense subgroups. If $G$ is profinite, then none of these contains an infinite relatively compact suitable set.

Proof. (i) This follows from the equation $d(G)=\log w(G)$ valid for any compact group $G$, see [3, (3.1)].

(ii) Let $G$ be a compact group of weight $\boldsymbol{\aleph}_{1}$. Then by (i) it has a countable dense subgroup $H$. Suppose that $G$ is profinite and $H$ has an infinite relatively compact suitable subset $S$. Then by Remark b we would have

$$
\aleph_{0}=\operatorname{card} H \geq w(H)=w(G)=\aleph_{1},
$$

a contradiction.

Now we show that every profinite group of uncountable weight is in $\mathcal{C}$.

Lemma 3.3. Let $G$ be a profinite group of uncountable weight and let $\aleph<w(G)$ be an infinite cardinal. Then there is a closed subgroup $H$ such that $w(H)=\boldsymbol{\aleph}$.

Proof. Let $T$ be a suitable subset of $G$ with card $T=w(G)$ according to Proposition 3.1. Then $T$ contains a subset $S$ of cardinality $\aleph$. We set $H=\overline{\langle S\rangle}$. Now $S$ is discrete in $H \backslash\{1\}$ since $T$ is discrete in $G \backslash\{1\}$. Hence $S$ is an infinite suitable subset of the profinite group $H$. Hence, by Lemma $3.2(\mathrm{~b}), w(H)=\operatorname{card}(S)=\aleph$ follows.

Corollary 3.4. An infinite profinite group contains an infinite compact metric subgroup.

Proof. Let $G$ be an infinite profinite group. If $w(G)=\boldsymbol{\aleph}_{0}$, then $G$ itself is metric. If $G$ has uncountable weight, then we apply Lemma 3.3 with $\aleph=\aleph_{0}$.

Now we are ready to prove that every compact group is in $\mathcal{C}$ which is the main portion of the following result:

Theorem 3.5. Let $\aleph$ be an infinite cardinal and let $G$ be a compact group such that $\boldsymbol{\aleph}<w(G)$. Then there is a closed subgroup $H$ such that $w(H)=\boldsymbol{\aleph}$, and if $G$ is connected, $H$ may be chosen normal and connected.

Proof. Let $G_{0}$ denote the identity component of $G$. The case that $w(G)=w\left(G_{0}\right)$ is handled in Proposition 2.2. So we assume $w\left(G_{0}\right)<w(G)$. By Lemma 2.1 we may assume that $G$ is totally disconnected, that is, profinite. Then Lemma 3.3 proves the assertion of the theorem. 
In particular, we have the following conclusion:

Corollary 3.6. Every infinite compact group contains an infinite closed metric subgroup.

Recall that by Corollary 1.6 we know that there is even a closed abelian metric subgroup. For a compact connected group $G$, Corollary 1.3 shows that for any infinite cardinal $\aleph \leq w(G)$ there is in fact a closed connected abelian subgroup of weight $\aleph$.

Problem. If $G$ is a compact group and $\aleph$ is an infinite cardinal $\leq w(G)$, is there a normal closed subgroup $H$ such that $w(H)=\aleph$ ?

The answer is affirmative if it is affirmative for profinite groups. A profinite group has weight $\boldsymbol{\aleph}$ iff its set of open-closed normal subgroups has cardinality $\mathbf{\aleph}$. This observation points in the direction of an affirmative answer to the problem.

\section{The weights of closed subgroups of a locally compact group}

Now we finish the proof of the Main Theorem by showing that every locally compact group is in $\mathcal{C}$. A first step is the following:

Lemma 4.1. Every locally compact pro-Lie group belongs to $€$.

Proof. Let $N$ be a compact normal subgroup of $G$ such that $G / N$ is a Lie group. Then $w(G / N)=\aleph_{0}$. We conclude $w(G)=w(N)$. We apply Theorem 3.5 to $N$ and obtain a closed subgroup of $N$ of weight $\aleph$.

A Hausdorff topological group $H$ is called almost connected if $H / H_{0}$ is compact. In Montgomery's and Zippin's classic [11] we note Lemma 2.3.1 on p. 54 and the Theorem on p. 175 and thus find

Lemma 4.2. Every locally compact group contains an open almost-connected pro-Lie group.

Lemma 4.3. Let $H$ denote an infinite open subgroup of a topological Hausdorff group $G$. Let $\mathcal{X}$ be a set of cosets $\mathrm{Hg}$ of $\mathrm{G}$ modulo $H$. Then

$$
w(\langle\bigcup \mathcal{X}\rangle)=\max \{w(H), \operatorname{card}(\mathcal{X})\}
$$

Proof. Abbreviate $\langle\bigcup \mathcal{X}\rangle$ by $K$. Then $w(H) \leq w(K)$ and $\operatorname{card}(\mathcal{X}) \leq w(K)$ and thus

$$
\max \{\operatorname{card}(\mathcal{X}), w(H)\} \leq w(K)
$$


In order to prove the reverse inequality, let $D$ be a dense subset of $H$ of cardinality $d(H)$. For each finite tuple $\mathscr{F}=\left(H g_{1}, H g_{2}, \ldots, H g_{n}\right) \in X^{n}, n \in \mathbb{N}$, the set $P_{\mathcal{F}} \stackrel{\text { def }}{=} H g_{1} H g_{2} \cdots H g_{n}$ has a dense subset $\Delta_{\mathcal{F}} \stackrel{\text { def }}{=} D g_{1} D g_{2} \cdots D g_{n}$ of cardinality $d(H)$ with the density of $H$. Then $K=\bigcup_{\mathscr{F}} P_{\mathscr{F}}$, with the union extended over the set of finite $n$-tuples $\mathscr{F}$, has a dense subset $\Delta=\bigcup_{\mathscr{F}} \Delta_{\mathcal{F}}$ whose cardinality is $\leq \operatorname{card} \mathcal{X} \cdot d(H) \leq \max \{\operatorname{card}(\mathcal{X}), w(H)\}$ (see [9, p. 620, Proposition 12.16 and its proof]). It follows that

$$
w(K) \leq \operatorname{card}(\Delta) \cdot w(H) \leq \max \{\operatorname{card}(\mathcal{X}), w(H)\} .
$$

Now (4.1) and (4.2) together prove the assertion.

The following conclusion now completes the proof of the Main Theorem:

Lemma 4.4. Let $G$ be a Hausdorff topological group having an open subgroup $H \in \mathcal{C}$. Then $G \in \mathcal{C}$.

Proof. Let $\boldsymbol{\aleph}_{0} \leq \boldsymbol{\aleph}<w(G)$; we have to find a closed subgroup $K$ of the group $G$ with $w(K)=\boldsymbol{\aleph}$. If $\boldsymbol{\aleph}<w(H)$, then there is a closed subgroup of $K$ such that $w(K)=\boldsymbol{\aleph}$ since $H \in \mathcal{C}$. If $\boldsymbol{\aleph}=w(H)$, then we take $K=H$. Finally, assume $w(H)<\aleph$. Then we pick a subset $\mathcal{X}$ of $G / H$ of cardinality $\aleph$. Then $K=\langle\bigcup \mathcal{X}\rangle$ has weight $\operatorname{card} \mathcal{X}=\boldsymbol{\aleph}$ by Lemma 4.3 .

The following conclusion now completes the proof of the Main Theorem:

Corollary 4.5. Every locally compact group belongs to $\mathcal{C}$.

Proof. If $\boldsymbol{\aleph}=w(G)=\boldsymbol{\aleph}_{0}$ there is nothing to prove. Assume $\boldsymbol{\aleph}_{0} \leq \boldsymbol{\aleph}<w(G)$; we have to find a closed subgroup $K$ of $G$ such that $w(K)=\aleph$. By Lemma 4.2, let $H$ be an open pro-Lie subgroup of $G$. By Lemma 4.1 we have $H \in \mathcal{C}$. Then $G \in \mathcal{C}$ by Lemma 4.4 .

Let $G$ be a topological group. Then the local weight (or character) $w_{\text {loc }}(G)$ is the smallest among the cardinals of neighbourhood bases at the neutral element. We say that a collection $\left\{K_{i}: i \in I\right\}$ is a compact cover of $G$ when $\bigcup_{i \in I} K_{i}=G$ and $K_{i}$ is compact for all $i \in I$. The compact covering number $\kappa(G)$ of $G$ is defined as the smallest of the cardinals of the members of the set of compact covers of $G$.

If $G$ is any infinite locally compact group, then $G$ contains an open almost-connected pro-Lie subgroup $H$ according to Lemma 4.2. Even an almost-connected pro-Lie group $H$ contains a maximal compact subgroup $C$ such that $H$ is homeomorphic to $\mathbb{R}^{n} \times C$ for a cardinal $n$, finite iff $H$ is locally compact (see [10]). 
Thus let $C$ be a maximal compact subgroup of $H$. Then

$$
G \text { is homeomorphic to } \mathbb{R}^{n} \times C \times G / H \text {. }
$$

One calls $n$ the (topological) rank, $\operatorname{Rank}(G)$, of $G$. Thus for a nondiscrete locally compact group $G$ we have

$$
\begin{aligned}
w_{\mathrm{loc}}(G) & =\max \left\{w_{\mathrm{loc}}\left(\mathbb{R}^{\operatorname{Rank}(G)}\right), w(C)\right\}, \\
\kappa(G) & =\max \left\{\kappa\left(\mathbb{R}^{\operatorname{Rank}(G)}\right),(G: H)\right\} .
\end{aligned}
$$

If $G$ is abelian, then $G \cong \mathbb{R}^{\operatorname{Rank}(G)} \times G_{c}$ with a direct factor $G_{c}$ having a compact open subgroup $C$ (see [9], Theorem 7.57). Thus

$$
\begin{aligned}
w_{\text {loc }}(G) & =\max \left\{w_{\text {loc }}\left(\mathbb{R}^{\operatorname{Rank}(G)}\right), w(C)\right\}, \\
\kappa(G) & =\max \left\{\kappa\left(\mathbb{R}^{\operatorname{Rank}(G)}\right),\left(G_{c}: C\right)\right\} .
\end{aligned}
$$

If we set $\Gamma=\hat{G}$, then we have $\Gamma \cong \mathbb{R}^{n} \times \hat{G}_{c}$ where $\hat{G}_{c}$ has the open subgroup $C^{\perp} \cong\left(G_{c} / C\right)^{\wedge}$ of $\hat{G}_{c}$ as the annihilator of $C$; moreover, $\hat{C} \cong \hat{G}_{c} / C^{\perp}$. It follows that then $w_{\text {loc }}(\Gamma)=\kappa(G)$ and $\kappa(\Gamma)=w_{\text {loc }}(G)$. In this sense, $w_{\text {loc }}$ and $\kappa$ are "dual" cardinals.

In view of our Main Theorem we may summarize:

Theorem 4.6 (The Local Weight Theorem for Locally-Compact Groups). For a nondiscrete locally compact group $G$ select any almost-connected open subgroup $H$ and any maximal compact subgroup $C$ of $H$. Then

(1) $w_{\mathrm{loc}}(G)=\max \left\{\boldsymbol{\aleph}_{0}, w(C)\right\}$.

(2) $w(G)=\max \left\{w_{\text {loc }}(G),(G: H)\right\}$.

(3) If $\operatorname{Rank}(G) \neq 0$, then $\kappa(G)=\max \left\{\aleph_{0},(G: H)\right\}$.

Proof. We know that $G$ and $\mathbb{R}^{\operatorname{Rank}(G)} \times C \times G / H$ are homeomorphic, where $G / H$ is a discrete space since $H$ is an open subgroup of $G$. Thus

$$
w_{\mathrm{loc}}(G)=w_{\mathrm{loc}}\left(\mathbb{R}^{\operatorname{Rank}(G)} \times C\right) .
$$

Since $G$ is nondiscrete, $w_{\text {loc }}(G) \geq \boldsymbol{\aleph}_{0}$. Thus (1) follows. Also,

$$
w(G)=w(H) \cdot w(G / H)=w_{\mathrm{loc}}(H) \cdot \operatorname{card}(G / H),
$$

yielding (2). Finally, assume that the topological rank $\operatorname{Rank}(G)$ of $G$ is nonzero. Then $\kappa(G) \geq \kappa(H)=\aleph_{0}$, and since $G$ is homeomorphic to $H \times G / H$, statement (3) follows. 
As a consequence of the Main Theorem and Theorem 4.6 above we have

Corollary 4.7. Let $G$ be a locally compact group containing infinite compact subgroups and let $\boldsymbol{\aleph}_{0} \leq \boldsymbol{\aleph}<w_{\text {loc }}(G)$. Then there is a compact subgroup of weight $\boldsymbol{\aleph}$.

Proof. Suppose that $H$ is an almost-connected open subgroup of $G$. Then $H$ is homeomorphic to $\mathbb{R}^{\operatorname{Rank}(G)} \times C$ for a maximal compact subgroup $C$ of $H$. If $K$ is any compact subgroup of $G$, then $K \cap H$ is open in $K$ and a conjugate of $K \cap H$ is contained in $C$ (see [10]). Thus, if $C$ is finite, then all compact subgroups of $G$ are finite. In that case

$$
w_{\mathrm{loc}}(G)=w_{\mathrm{loc}}(H)=\aleph_{0}
$$

by (1). Otherwise, $w_{\text {loc }}(G)=w(C)$. Thus $\boldsymbol{\aleph}_{0} \leq \boldsymbol{\aleph}<w(C)$ and so the Main Theorem yields a compact subgroup of $C$ of weight $\aleph$.

\section{An application of the Main Theorem}

Let $(G, \tau)$ be an arbitrary abelian topological group. We shall continue to write abelian groups additively, unless specified otherwise such as in the case of the multiplicative circle group $\mathbb{S}^{1}=\{z \in \mathbb{C}:|z|=1\}$. A character on $(G, \tau)$ is a continuous morphism $\chi: G \rightarrow \mathbb{T}=\mathbb{R} / \mathbb{Z}$. The pointwise sum of two characters is again a character, and the set $\widehat{G}$ of all characters is a group with pointwise addition as the composition law. If $\widehat{G}$ is equipped with the compact open topology $\widehat{\tau}$, it becomes a topological group $(\widehat{G}, \widehat{\tau})$ which is called the dual group of $(G, \tau)$.

Definition 5.1 (Varopoulos). Let $G$ and $H$ be two abelian groups. We say that $G$ and $H$ are in duality if and only if there is a $\mathbb{Z}$-bilinear function

$$
\langle\cdot, \cdot\rangle: G \times H \longrightarrow \mathbb{T}=\mathbb{R} / \mathbb{Z}
$$

such that

$$
\begin{array}{ll}
\left(\forall 0_{G} \neq g \in G\right)(\exists h \in H) \quad\langle g, h\rangle \neq 0_{\mathbb{T}}, \\
\left(\forall 0_{H} \neq h \in H\right)(\exists g \in G) \quad\langle g, h\rangle \neq 0_{\mathbb{T}} .
\end{array}
$$

Definition 5.2 (Varopoulos). Assume that $G$ and $H$ are in duality. Then a topology $\tau$ on $G$ is compatible with the duality if $(G, \tau)^{\wedge}=H$.

Typically, if $G$ is a locally compact abelian group and $\hat{G}$ its Pontryagin dual, that is, its character group, then $G$ and $\hat{G}$ are in duality, but there are group topologies $\tau$ on $G$ which are necessarily coarser than the given locally compact topology on $G$ but which are nevertheless compatible with this duality (see [2]). One of the best 
known is the so-called Bohr topology $\tau^{+}$on $G$ which is the topology of pointwise convergence when $G$ is considered as the group of characters of $\hat{G}$ via Pontryagin duality. By its very definition, the Bohr topology $\tau^{+}$is a precompact topology. Indeed, a topological group $G$ is said to be precompact or totally bounded if for any neighborhood $U$ of the neutral element in $G$, there is a subset $F \subseteq G$ with $\operatorname{card}(F)<\boldsymbol{\aleph}_{0}$ such that $F U=G$ (see [3] for general properties of precompact groups).

Next we need to generalize the concept of total boundedness:

Definition 5.3. Let $\aleph$ be a cardinal number. A topological group $G$ is said to be $\aleph$-bounded when for every neighborhood $U$ of the neutral element in $G$, there is a subset $S \subseteq G$ with $\operatorname{card}(S)<\aleph$ such that $S U=G$.

According to this definition, a group $G$ is totally bounded iff it is $\boldsymbol{\aleph}_{0}$-bounded. It is uniformly Lindelöf if it is $\boldsymbol{\aleph}_{1}$-bounded.

From Theorem 3.4 in [4] we have the following information:

Let $X$ be a set, let $M$ be a metrizable space, and let $Y$ be a subset of $M^{X}$ that is equipped with some bornology $\mathscr{B}$ consisting of pointwise relatively compact sets (that is, $\mathscr{B}$ is a family of pointwise relatively compact subsets of $Y$ which is closed under taking subsets and unions of finitely many elements, and contains all finite subsets of $X$ ). Denote by $\mu_{\mathscr{B}}$ the uniformity on $Y$ defined as $\sup \left\{\mu_{F}: F \in \mathscr{B}\right\}$. The concept of an $\boldsymbol{\aleph}$-bounded topological group generalizes rather immediately to that of an $\boldsymbol{\aleph}$-bounded uniform space. Now we have

The $\aleph$-Boundedness Theorem. If $\boldsymbol{\aleph}$ is a cardinal such that $w(M)<\boldsymbol{\aleph}$, then the following statements are equivalent:

(i) $(\forall F \in \mathscr{B}) w(F)<\boldsymbol{\aleph}$.

(ii) $\left(X, \mu_{\mathscr{B}}\right)$ is $\aleph$-bounded.

For a topological abelian group $\Gamma$ and a cardinal $\aleph$ we let $\mathcal{K}_{\aleph}(\Gamma)$ denote the set of all compact subsets $K \subseteq \Gamma$ with $w(K)<\aleph$.

Lemma 5.4. Let $\Gamma$ be the character group of an abelian topological group $G$. Let $\boldsymbol{\aleph}$ be a cardinal $\leq w(G)$, and let $\Gamma$ have the topology of uniform convergence on compact subsets $K \in \mathcal{K}_{\boldsymbol{\aleph}}(G)$. Then $\Gamma$ is $\aleph$-bounded.

Proof. We apply the $\aleph$-Boundedness Theorem with $\Gamma=X, \mathbb{T}=M, G=Y$, $\mathcal{K}_{\mathbb{N}}(\Gamma)=\mathscr{B}$, and, finally, with the topology of uniform convergence on sets of $\mathcal{K}_{\aleph}(\Gamma)$ being the uniform topology of $\mu_{\mathcal{B}}$. We have $w(M)=w(\mathbb{T})=\aleph_{0} \leq \aleph$. Hence the implication (i) $\Rightarrow$ (ii) of the $\boldsymbol{\aleph}$-Boundedness Theorem yields the assertion of the lemma. 
We now discuss some facts about the Bohr topology in the next example for the sake of completeness and because it plays a role in our subsequent discussion which, at least in the case that $G$ is discrete, provides a substantial cardinality of topologies on $G$ which are compatible with the Pontryagin duality of $G$.

Facts 5.5. Let $G=(A, \tau)$ be a locally compact abelian group and denote by $G^{\alpha}$ the Bohr compactification of $G$. One may think of $G^{\alpha}$ as the compact group which is the dual of $\hat{G}$ when the latter is equipped with the discrete topology. Let $\eta: G \rightarrow G^{\alpha}$ be the Bohr compactification morphism and let $\tau^{+}$be the Bohr topology on $G$. Equivalently, $\tau^{+}$is the pull-back topology on $A$ (that is, the topology which makes $\eta$ an embedding $\left.\eta: G^{+} \rightarrow G^{\alpha}\right)$. We write $G^{+}$for the topological group $\left(A, \tau^{+}\right)$. Then:

(i) If $K$ is a $\tau^{+}$-compact subset of $A$, then $K$ is $\tau$-compact.

(ii) $\tau^{+}$is compatible with the duality between $A$ and $\hat{G}$, that is, $\hat{G^{+}}=\hat{G}$. Also, $\hat{G^{+}}=\left(G^{\alpha}\right)^{\wedge}$ (algebraically).

(iii) If $\sigma$ is any group topology on $A$ such that $\tau^{+} \subseteq \sigma \subseteq \tau$, then $\sigma$ is compatible with the duality between $A$ and $\hat{G}$.

(iv) $w(G)=w(\hat{G}), w\left(G^{+}\right)=w\left(G^{\alpha}\right)=\operatorname{card}(\hat{G})$.

Proof. For easy reference we provide proofs.

(i) This is Glicksberg's theorem [5].

(ii) This a consequence of the universal property of the Bohr compactification (see $[3,4.26])$.

(iii) Every character $\chi \in \hat{G}, \chi: G=(A, \tau) \rightarrow \mathbb{T}$ is $\tau^{+}$-continuous by (ii). Then it is $\sigma$-continuous since $\tau^{+} \subseteq \sigma$. Conversely, let $\chi:(A, \sigma) \rightarrow \mathbb{T}$ be a $\sigma$-continuous character, then it is $\tau$-continuous, since $\sigma \subseteq \tau$. Hence a character $A \rightarrow \mathbb{T}$ is $\sigma$-continuous iff it is $\tau$ continuous.

(iv) For the equalities $w(G)=w(\hat{G})$ and $w\left(G^{\alpha}\right)=\operatorname{card}\left(G^{\alpha}\right)^{\wedge}$ see e.g. [9, Theorem 7.76 (i) and (ii), p. 364]. Since $G^{+}$has a dense homeomorphic image in $G^{\alpha}$, we have $w\left(G^{+}\right)=w\left(G^{\alpha}\right)$. Now $\left(G^{\alpha}\right)^{\wedge}=\hat{G}$ by (ii) above. This proves the assertion.

Proposition 5.6. Let $G$ be a locally compact abelian group. Let $H$ be a first countable subgroup of $G^{+}$. Then its closure $\mathrm{cl}_{G} H$ in $G$ is compact.

In particular, if $G$ has no nonsingleton compact subgroups, such as $G=\mathbb{Z}$ or $G=\mathbb{R}$, then the precompact group $G^{+}$has no nonsingleton metric subgroups.

Proof. We continue the notation of Facts 5.5. Let $H$ be a first countable nonsingleton subgroup of $G^{+}$. Then $H$ is $\tau^{+}$-precompact since $G^{+}$is precompact, and 
its topology is determined by its convergent sequences since it is first countable. On the other hand, by Facts 5.5 (i), $H$ has the same convergent sequences for $\tau$ and $\tau^{+}$. Hence, both topologies coincide on $H$. Since $H$ is $\tau$-precompact, the locally compact group $\mathrm{cl}_{G} H$ is $\tau$-precompact and thus is compact.

Now assume that $G$ is a locally compact abelian group without compact nondegenerate subgroups (such as $G=\mathbb{Z}$ or $G=\mathbb{R}$ ). Then $\mathrm{cl}_{G} H$ is singleton, and so is $H$.

Consider now a locally compact abelian group $G$ with dual group $\Gamma$. If $w_{\text {loc }}(\Gamma)$ is uncountable, then by Theorem 4.6, $\Gamma$ contains a compact subgroup $C_{\Gamma}$ with weight $w\left(C_{\Gamma}\right)=w_{\text {loc }}(\Gamma)$. If $w_{\text {loc }}(\Gamma)=\aleph_{0}$, then there always exist infinite metrizable compact subsets of $\Gamma$. Now let $\boldsymbol{\aleph} \leq \kappa(G)=w_{\text {loc }}(\Gamma)$ be any infinite cardinal. Consider $G$ as the character group of the locally compact group $\Gamma$.

Write $\tau_{\aleph}$ for the topology of uniform convergence on the sets $K \in \mathcal{K}_{\aleph}(\Gamma)$. This topology is locally quasiconvex by construction. For the definition of local quasiconvexity we refer to [1] where one finds a comprehensive study of locallyquasiconvex groups introduced by Vilenkin; we are not using this notion except for reporting that some group topologies we shall encounter are of this type. We remark that the topology $\tau_{\mathbf{N}_{0}}$ coincides with the Bohr topology $\tau^{+}$introduced previously. We have all abelian topological groups $\left(G, \tau_{\boldsymbol{\aleph}_{\alpha}}\right), 0 \leq \alpha \in \Omega$, are locally quasiconvex.

The interval $\left[\boldsymbol{\aleph}_{0}, w_{\text {loc }}(\Gamma)\right]$ of all infinite cardinals up to and including the local weight of $\Gamma$ is well-ordered. Let $\Omega$ the initial set of ordinals $0<1<\cdots \leq \xi$ which is order isomorphic to $\left[\boldsymbol{\aleph}_{0}, w_{\text {loc }}(\Gamma)\right]$.

It is in the proof of the following lemma that we use the principal result of this paper.

Main Lemma 5.7. Let $G$ be a noncompact, locally compact abelian group with dual group $\Gamma$. For $\alpha<\xi$ in $\Omega$, the following conclusions hold:

(i) $\mathcal{K}_{\boldsymbol{\aleph}_{\alpha}}(\boldsymbol{\Gamma}) \subsetneq \mathcal{K}_{\aleph_{\alpha+1}}(\boldsymbol{\Gamma})$,

(ii) $\tau_{\aleph_{\alpha}} \subsetneq \tau_{\aleph_{\alpha+1}}$.

Proof. If $G$ is metrizable, then $\tau_{\boldsymbol{\aleph}_{0}}<\tau_{\boldsymbol{\aleph}_{1}}$ because the former topology is precompact and the latter coincides with the original noncompact, locally compact topology of $G$. Similarly,

$$
\mathcal{K}_{\aleph_{0}}(\Gamma) \subsetneq \mathcal{K}_{\aleph_{1}}(\Gamma)
$$

because $\Gamma$ contains infinite compact subsets. Therefore, we may assume without loss of generality that $w_{\text {loc }}(G)>\boldsymbol{\aleph}_{0}$.

(i) If $\alpha=0$, then we must verify that $\mathcal{K}_{\aleph_{0}} \subsetneq \mathcal{K}_{\aleph_{1}}$. But this is clear because $\Gamma$ is a nondiscrete locally compact abelian group and, by Corollary $4.7, \Gamma$ and must 
contain a metrizable compact subgroup or a copy of $\mathbb{R}$. In either case, it contains infinite metrizable compact subsets.

So, assume that $\alpha>0$. By the Main Theorem (indeed by Theorem 3.5), the compact group $C_{\Gamma}$ contains a subgroup $K$ of weight $w(K)=\boldsymbol{\aleph}_{\alpha}$. What is relevant for the proof is the fact that $\Gamma$ contains a compact subspace $K$ of weight $\boldsymbol{\aleph}_{\alpha}$. Thus $K \in \mathcal{K}_{\boldsymbol{\aleph}_{\alpha+1}} \backslash \mathcal{K}_{\boldsymbol{\aleph}_{\alpha}}$. The inclusion $\mathcal{K}_{\boldsymbol{\aleph}_{\alpha}}(\Gamma) \subseteq \mathcal{K}_{\boldsymbol{\aleph}_{\alpha+1}}(\Gamma)$ is trivial.

(ii) From statement (i) it follows that $\tau_{\boldsymbol{\aleph}_{\alpha}} \subseteq \tau_{\boldsymbol{\aleph}_{\alpha+1}}$. From Lemma 5.4 we know that $\left(G, \tau_{\boldsymbol{\aleph}_{\alpha}}\right)$ is $\boldsymbol{\aleph}_{\alpha}$-bounded and that $\left(G, \tau_{\boldsymbol{\aleph}_{\alpha+1}}\right)$ is $\boldsymbol{\aleph}_{\alpha+1}$-bounded. Suppose, by way of contradiction, that the topologies $\tau_{\boldsymbol{\aleph}_{\alpha}}$ and $\tau_{\boldsymbol{\aleph}_{\alpha+1}}$ were equal. Then the implication (ii) $\Rightarrow$ (i) of the $\aleph$-Boundedness Theorem would imply that every compact subset $K \subseteq \Gamma$ of weight $w(K)<\boldsymbol{\aleph}_{\alpha+1}$ would have weight $w(K)<\boldsymbol{\aleph}_{\alpha}$. This would contradict (i) above, and this contradiction shows that $\tau_{\boldsymbol{\aleph}_{\alpha+1}}$ is strictly finer than $\tau_{\aleph_{\alpha}}$.

The Main Lemma 5.7 establishes the essential result of this section:

Theorem 5.8. Let $(G, \tau)$ be a locally compact abelian group which has an infinite compact covering number $\kappa(G)$. Then

(i) for each cardinal $\boldsymbol{\aleph}$ with $\boldsymbol{\aleph}_{0} \leq \boldsymbol{\aleph} \leq \kappa(G)$ there exists a locally-quasiconvex group topology $\tau_{\aleph}$ on $G$ such that $\left(G, \tau_{\aleph}\right)^{\wedge}=\hat{G}$, and

(ii) for $\boldsymbol{\aleph}_{0} \leq \boldsymbol{\aleph}<\boldsymbol{\aleph}^{\prime} \leq \kappa(G)$ one has $\tau_{\boldsymbol{\aleph}} \subsetneq \tau_{\boldsymbol{\aleph}^{\prime}} \subseteq \tau$.

Thus, if $\left[\boldsymbol{\aleph}_{0}, \kappa(G)\right]$ denotes the full interval of infinite cardinals up to $\kappa(G)$, then Theorem 5.8 provides card $\left[\aleph_{0}, \kappa(G)\right]$-many locally-quasiconvex group topologies on the abelian group $G$ all of which are coarser than the original topology of $G$ and yield the (locally compact abelian) group $\hat{G}$ as character group. Indeed, since $\tau_{\aleph_{0}} \leq \tau_{\aleph} \leq \tau$, it follows that $\left(G, \tau_{\aleph}\right)^{\wedge}=\hat{G}$ algebraically. Furthermore, since $\tau_{\aleph_{0}}$ is the Bohr topology and the compact subsets of $\tau_{\aleph_{0}}$ and $\tau$ coincide by Glicksberg's theorem [5], this also holds for any other topology $\tau_{\aleph}$.

Corollary 5.9. Let $G$ be an infinite discrete abelian group. Then

(i) for each cardinal $\boldsymbol{\aleph}$ with $\boldsymbol{\aleph}_{0} \leq \boldsymbol{\aleph} \leq \operatorname{card}(G)$ there is a locally-quasiconvex group topology $\tau_{\aleph}$ on $G$ such that $\left(G, \tau_{\aleph}\right)^{\wedge}=\hat{G}$, and

(ii) for $\boldsymbol{\aleph}_{0} \leq \boldsymbol{\aleph}<\boldsymbol{\aleph}^{\prime} \leq \operatorname{card}(G)$ one has $\tau_{\boldsymbol{\aleph}} \subsetneq \tau_{\boldsymbol{\aleph}^{\prime}}$.

Again, if $\left[\boldsymbol{\aleph}_{0}, \operatorname{card}(G)\right]$ denotes the full interval of infinite cardinals up to the cardinality of $\operatorname{card}(G)$, then Corollary 5.9 above provides $\operatorname{card}\left[\boldsymbol{\aleph}_{0}, \operatorname{card}(G)\right]$-many locally-quasiconvex group topologies $\tau$ on the abelian group $G$ all of which yield the (compact abelian) group $\hat{G}$ as character group of $(G, \tau)$. 
Acknowledgments. We express our gratitude to an extremely thorough and helpful referee who not only assisted us in avoiding a sequence of errors of a typographical sort but also suggested a certain reorganisation of our material.

\section{Bibliography}

[1] W. Banaszczyk, Additive Subgroups of Topological Vector Spaces, Lecture Notes in Mathematics 1466, Springer-Verlag, Berlin, 1991.

[2] M. J. Chasco, E. Martín-Peinador and V. Tarieladze, On Mackey topology for groups, Studia Math. 132 (1999), 257-284.

[3] W. W. Comfort, Topological groups, in: Handbook of Set-Theoretic Topology, NorthHolland, Amsterdam (1984), 1143-1263.

[4] M. V. Ferrer and S. Hernández, Dual topologies on non-abelian groups, Topology Appl., to appear.

[5] I. Glicksberg, Uniform boundedness for groups, Canad. J. Math. 14 (1962), 269276.

[6] S. Hernández, Questions raised at the conference "Algebra meets Topology," Barcelona, July 2010, hernande@mat.uji.es.

[7] E. Hewitt and K. A. Ross, Abstract Harmonic Analysis I, Grundlehren der Mathematischen Wissenschaften 115, Springer-Verlag, Berlin, 1963.

[8] K. H. Hofmann and S. A. Morris, A structure theorem on compact groups, Math. Proc. Cambridge Philos. Soc. 130 (2001), 409-426.

[9] K. H. Hofmann and S. A. Morris, The Structure of Compact Groups, Second edition, Walter de Gruyter, Berlin, 2006.

[10] K. H. Hofmann and S. A. Morris, The structure of almost connected pro-Lie groups, J. Lie Theory 21 (2011), 347-383.

[11] D. Montgomery and L. Zippin, Topological Transformation Groups, Second edition, Robert E. Krieger Publishing, Huntington, 1966.

[12] L. Ribes and P. Zaleskii, Profinite Groups, Second edition, Springer-Verlag, Berlin, 2010.

[13] W. R. Scott, The number of subgroups of given index in a nondenumerable group, Proc. Amer. Math. Soc. 5 (1954), 19-22.

[14] E. A. Walker, Subdirect sums and infinite abelian groups, Pacific J. Math. 9 (1959), 287-291.

[15] E. I. Zelmanov, On periodic compact groups, Israel J. Math. 77 (1992), 83-95. 
Received January 14, 2012; revised April 6, 2012.

\section{Author information}

Salvador Hernández, Universitat Jaume I, INIT and Depto de Matemáticas, Campus de Riu Sec, 12071 Castellón, Spain.

E-mail: hernande@mat.uji.es

Karl H. Hofmann, Fachbereich Mathematik, Technische Universität Darmstadt, Schlossgartenstraße 7, 64289 Darmstadt, Germany.

E-mail: hofmann@mathematik.tu-darmstadt.de

Sidney A. Morris, School of Science, IT, and Engineering, University of Ballarat, Victoria 3353, Australia;

and School of Engineering and Mathematical Sciences, La Trobe University, Bundoora, Victoria 3086, Australia.

E-mail: morris.sidney@gmail.com 